\title{
The design and research of the shared kitchen system in the hospital
}

\author{
YE Xin ${ }^{1}$, JI Qian ${ }^{1, a}$ \\ ${ }^{1}$ Huazhong University of Science and Technology,430074 Wuhan
}

\begin{abstract}
The shared economy has been developing rapidly with low cost, low consumption and high environmental efficiency features under the background of internet waves. The shared economy model has emerged in housing, catering, and travel. As people look forward to high quality of life and their social interaction need, the shared kitchen platform arises at the right moment. This paper takes the shared kitchen as an example, focusing on the patients and their caregivers, the existing shared kitchens and its service platform as well as the space system design and human-computer interaction of the shared kitchen have been investigated and analysized. Taking the "high efficiency, the intelligence and the humanization" as the design principles, we are committed to exploring new directions for modular kitchen design under the background of shared economy.
\end{abstract}

\section{The purpose and significance of research}

There are four main dining types for patients in hospital: taking meals in a hospital or a restaurant, ordering takeout food and delivered by caregivers, while most patients would prefer the last two choices other than taking meals in a hospital or a restaurant.

The reasons for this phenomenon are as following: Firstly, the food in the hospital canteen does not meet with their taste. Secondly, caregivers are concerned about the freshness of the food as well as the clean cooking process. Thirdly, the price of the food in restaurant is relatively high, the service attitude and the environment would be poor in some cheaper canteens. ${ }^{[1]}$

Family meal delivery has become the most common way for patients and caregivers, however, through longterm observation and research, we find that it is difficult for those who live far away from hospital to enjoy family meal delivery. Therefore, this paper aims to propose solutions to the problems, which will not only meet the patients' needs of eating healthy food, but also not too much interruption of caregivers' daily life and work. It will also be able to dig out new commercial interests in line with the trend of economic development.

\section{Preliminary investigation and analysis}

Our objectives of investigation refer to inpatients, caregivers, and nursing staff.

For patients, hospitalization often brings some psychological pressure to them, so they are prone to have the feeling of anxiety, loneliness, fear, depression and pessimism, they hope that caregivers can give more care and companionship. At this time, family's help and care can effectively enhance the patient's confidence in receiving treatment and help improve the patient's quality of life.

In addition, for caregivers, it is found that they are also prone to feel anxious, tense, sad, and self-blamed, delivery meals will also increase the trouble in their lives. through on-site observation and investigation, we notice that there are 10 steps for the caregivers to cook and deliver meals to patients. (table 1)

Table 1. caregiver process analysis

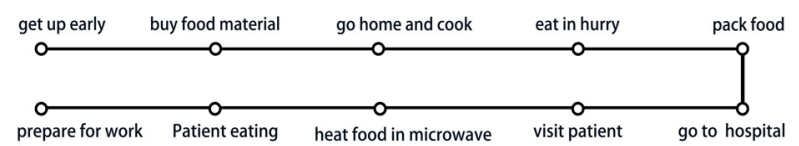

First of all, getting up early can easily lead to the lack of sleep and increase fatigue. Secondly, buy ingredients then go home cooking, caregivers should spend too much time on the preparing and cooking. Thirdly, the caregivers always finish the meal quickly to save time and then pack the food to the hospital, which will easily cause irregular diet and some healthy problems. Fourthly, packaged food will not provide the atmosphere of eating at home. Fifthly, traffic jam at rush hour will waste time. Sixthly, the packaged food always needs heating, but reheating will affect the taste of the food. Finally, caregivers will feel exhausted after the whole process. Many of these steps have negative impacts on caregiver's physical and

*Corresponding author: ${ }^{\mathrm{a}}$ author@e-mail.org 
mental health, so problems can be revealed through the observation and analysis.

\subsection{The development of shared kitchens and the concept of new shared kitchens}

\subsubsection{Existing shared kitchen platform research}

The existing common kitchens are operated meeting the needs of two types of people, office workers who want to eat homemade dish and people who want to earn money through cooking skills. The representative shared kitchen platforms are two brands "I have a meal " and "Go home for dinner."

"I have a meal" belongs to the social sharing platform. It discovers the resources of the dinner in the city, regularly publishes dinner information, gathers customers together. It embodies the "Kitchen + Social" marketing model. The target group is the people who love food.

"Go home for dinner " is the "personal sentiment" style type. Its marketing mode is private kitchen cooking - delivery service - dining. The target group is the person who likes cooking and the office workers who are looking for " hometown taste". ${ }^{[8]}$

\subsubsection{A shared kitchen design proposed in this paper}

This paper puts forward a new direction for the development of shared kitchen platform in hospital. With "kitchen + health" as its marketing model, the target customers are patients who expect to recuperate health through diet and caregivers who want to spend more time with patients. The new type of shared kitchen advocates users to control the freshness of food ingredients, as well as food hygiene and safety, let the patient eat health.

As we all know, diet therapy plays an important role in restoring health for the patient. Due to the difference of the physical condition, patients' needs for food and nutrition are different. But caregivers often know very little about the practice and efficacy of food for patient. Previous research has found that there are few products with the function of healthy dietary guidance in the market, while patients' need for dietary therapy is very huge.

The new shared kitchen takes "dietotherapy" as an innovative point. By analyzing the diagnostic reports given by the hospital, the intelligent machine collate the trace elements and substances required by the patients. Based on the principles of comprehensive diversification, nutrition and equalization, specific recipes and diet matching are given. Caregivers can easily cope with the problem of "what to eat today".

\subsection{Advantage and innovation of new shared kitchen}

\subsubsection{Food safety}

In the operating mode of the new shared kitchen, users make an appointment to the shared kitchen through the hospital health card. The system intelligently analyses the patient's health status, give a variety of recipes for caregivers to select. And user can view the original material of food needed to be prepared. From food ingredients to production, all the process will be checked by users to ensure the safety of food.

\subsubsection{The credit system of real name authentication}

According to the survey, almost all hospitals have implemented real-name registration.

Compared with the existing shared kitchen platform which lacks credit system among customers, the new shared kitchen is bound to the user through the hospital health card, so it is easy to manage and maintain.

\subsubsection{Healthy diet guidance as a marketing point}

The existing shared kitchen platform does not Satisfy the rigid demand of the customers, but has grasped the characteristics of the customer with the "feeling" meal, the customer stickiness is not high ${ }^{[8]}$. It is easy to encounter bottlenecks in development.

The new shared kitchens focusing on "healthy eating," especially in hospitals, is urgent for patients to adjust their functions by using the characteristics of food. Therefore, dietary care and guidance are widely needed.

\section{Related human-machine dimension research, spatial color and layout, and product $\mathrm{HCl}$ mode analysis}

At present, most of the hospitals in China have limited environmental space, the whole decorated kitchen with large equipment and large area is not suitable for the shared kitchen in hospitals. This paper investigates and analyses the application of ergonomic parameters in kitchens and cabinets. Considered color matching, function partitioning and human-computer interaction, we propose a modular of future shared 
kitchen design scheme with small space and complete functions from the actual environment.

\subsection{Study of man-machine size}

According to the operation basis of ergonomics, we determined the dimensions of the cabinet plane and section to meet the user's operational requirements. The comfort data of different heights were obtained from the literature research as follows: ${ }^{[5]}$ (table 2)

Table 2. Corresponding comfort heights

\begin{tabular}{|l|l|l|l|l|l|l|l|l|l|}
\hline height & $150 \mathrm{~cm}$ & $153 \mathrm{~cm}$ & $155 \mathrm{~cm}$ & $158 \mathrm{~cm}$ & $160 \mathrm{~cm}$ & $163 \mathrm{~cm}$ & $165 \mathrm{~cm}$ & $168 \mathrm{~cm}$ & $170 \mathrm{~cm}$ \\
\hline $\begin{array}{l}\text { Comfortable } \\
\text { operating height }\end{array}$ & $79 \mathrm{~cm}$ & $80 \mathrm{~cm}$ & $81.5 \mathrm{~cm}$ & $83 \mathrm{~cm}$ & $84 \mathrm{~cm}$ & $85.7 \mathrm{~cm}$ & $86.5 \mathrm{~cm}$ & $88 \mathrm{~cm}$ & $89 \mathrm{~cm}$ \\
\hline
\end{tabular}

At present, the height of most operating platform is $860 \mathrm{~mm}$. According to the national scale of the human body scale, the 10th percentile of the adult female height is $150.3 \mathrm{~cm}$, and the 90th percentile of the adult female height is $164 \mathrm{~cm}$. Therefore, the height of the corresponding console is from $79 \mathrm{~cm}$ to $86.5 \mathrm{~m}$ [5] (Figure 1)
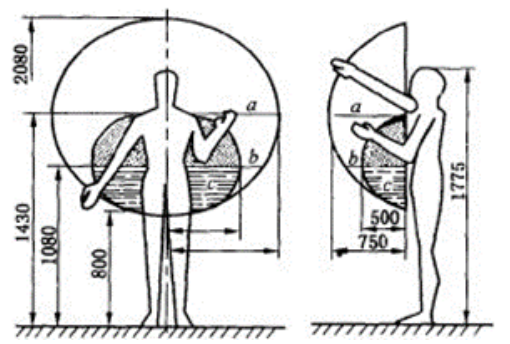

Figure 1. the height for standing position

The width of a person in standing operation is $660 \mathrm{~mm}$, and the male is $700 \mathrm{~mm}$. In addition, the size correction should also be considered. Through manmachine data analysis, we know that when the angle between the arm and the body is 15 degrees, the work is relatively easy. So the width of the operation surface should be $760 \mathrm{~mm}$. And the width of the console should be greater than or equal to $760 \mathrm{~mm}$ if conditions permit. ${ }^{[5]}$ (Figure 2)

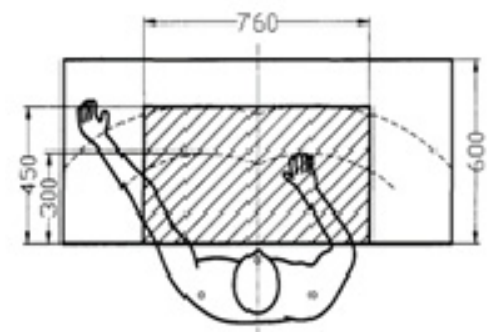

Figure 2. the width of operating platform for standing position

\subsection{Color collocation and research}

Color matching has a certain effect on visual perception and space size ${ }^{[4]}$. Generally speaking, highbrightness colors have the characteristic of retreat, it can make the space look more spacious. Moreover, under the pressure of tension, warm and bright environment can give people some psychological support and comfort.

\subsection{Functional partition and layout}

Under normal circumstances, the kitchen is mainly divided into four major areas: operating area, washing area, cooking area and storage area. Because the use environment is in the hospital, and user has uncertainty, the material does not need to be stored. Therefore, the main functional areas can be divided into three parts: operation area, washing area and cooking area.

There are four common kitchen layouts, One-line layout, L-type, U-type layouts, and island-type layouts. Among these layouts, the L-shaped layout is also called a semi-enclosed layout. Since a triangular area is formed between the washing area, the operation area and the cooking area, user can make full use of the blue triangle area shown in figure 3. So it is a more practical and space-saving kitchen layout design. [7] (figure 3)
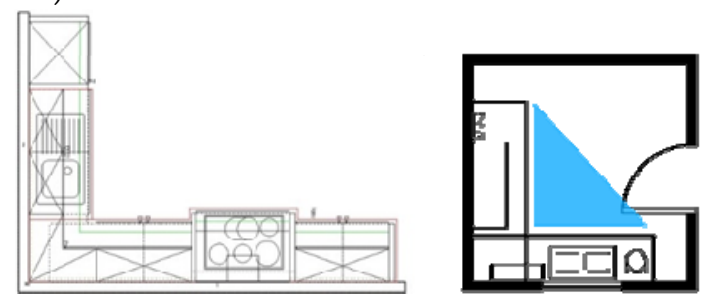

Figure 3. Layout and design of L type kitchen

\section{Design scheme}

The design of shared kitchen system should not only focus on space layout planning, but also consider the interaction between users and products in the pre use, use, and post - use stages. Make the product easy to use by simplifying the operation. (Figure 4)

User experience is embodied in three aspects: form, content and behavior. The form is the appearance of the kitchen and the interface of the interactive graphics; the content is shown on the function and information of the product, and the behavior refers to the human-computer interaction ${ }^{[6]}$. In the design process, we need to focus on the specific form and interaction model of the product in order to enhance the user experience. ${ }^{[6]}$

Before use: user is bound to the new shared kitchen platform on the mobile phone. According to the medical record information and the inspection 
report, system provides a number of recipes guidance for the recovery of the body function. User can view the corresponding list of Food material after selecting a recipe.

In use: After the user makes an appointment for the free kitchen, the smart door lock has a verification code, user unlocks the shared kitchen through the verification code. Then the system starts charging, and the application guides the user to cook until the end of use.

After use: cleaning is the most important problem that public facilities need to pay attention to. Especially in the hospital, besides the basic cleanliness, it must be sterilizing. Therefore, after the end of the use, system reminds the user to clean, and it ends the billing until the cleaning is finished.

In addition to the ultraviolet bactericidal in the kitchen, the wavelength $240-270 \mathrm{~nm}$ ultraviolet rays can destroy the DNA and RNA of the bacteria within the $1.2 \mathrm{M}$, and make it lose the reproduction ability and achieve the purpose of the death of the disease bacteria. And the UV specific wavelengths can make the air chemical reaction to produce ozone that can destroy the protein of the pathogen. This disinfection method can kill the hepatitis virus, Escherichia coli and other daily bacteria, effectively ensuring the cleanliness and hygiene of the kitchen.

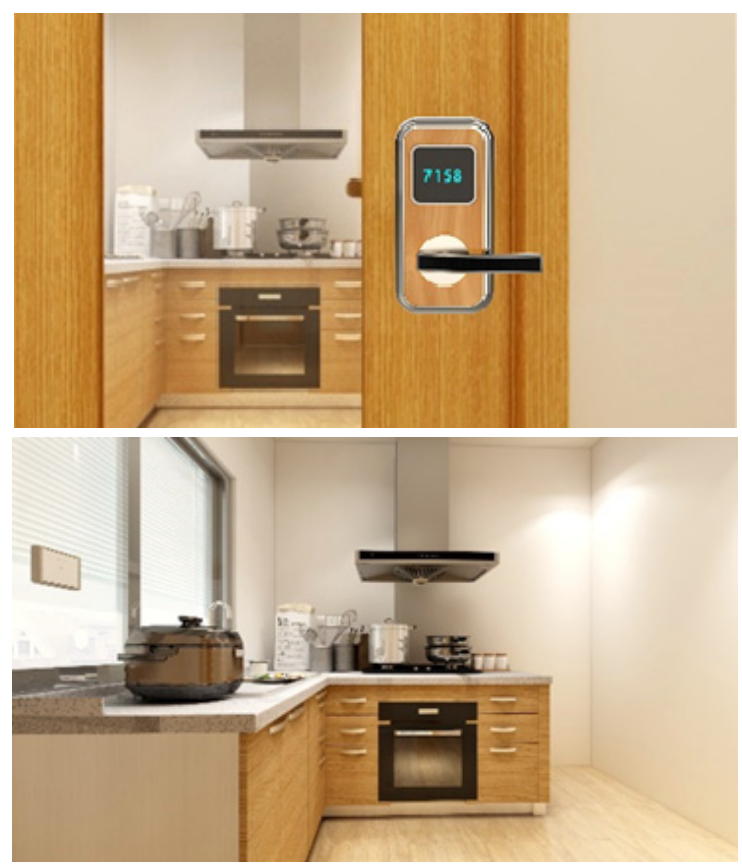

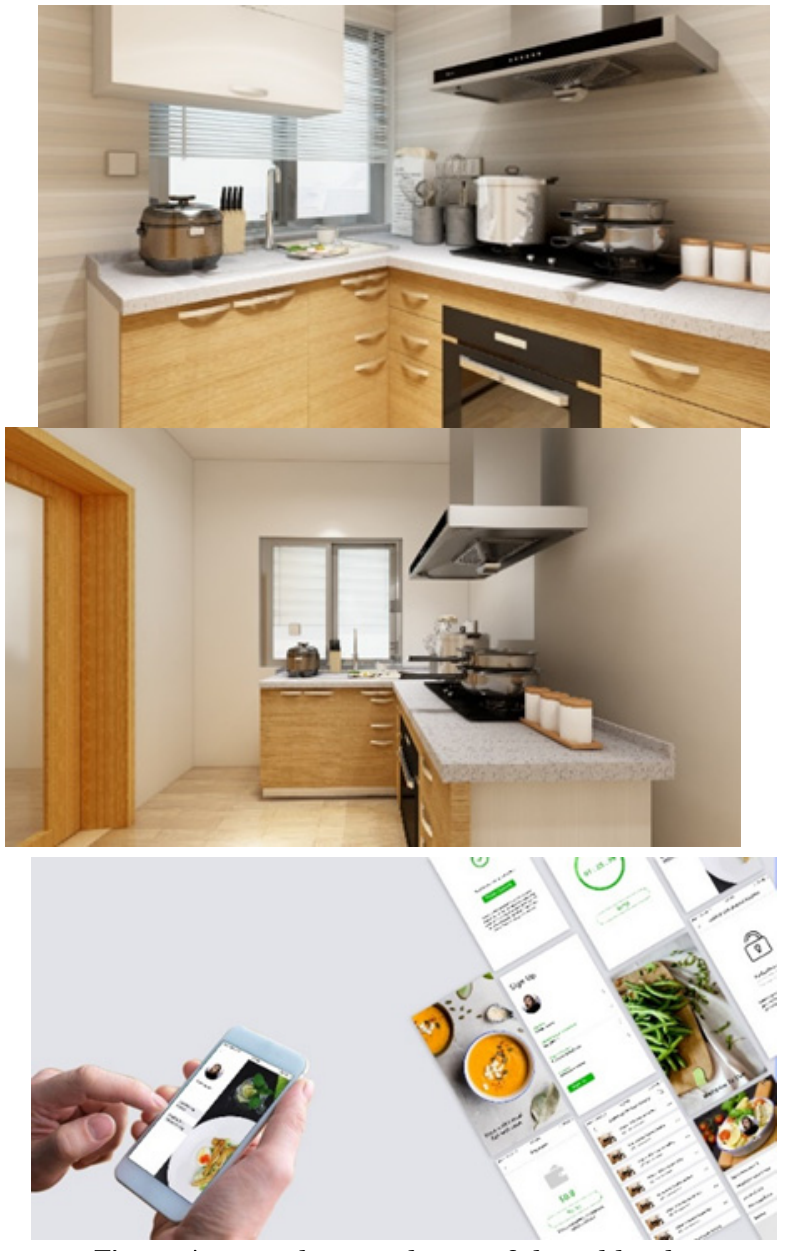

Figure 4. New design scheme of shared kitchen

\section{Concluding remarks}

With the development of information age, intelligent technology has been widely used in household kitchen and toliet. The new way of interaction and experience brought by technological progress has become the key factor to enhance the competitiveness of products. With the advancement of science and technology, the sharing of kitchen systems in the future will be able to satisfy people's increasingly high-level needs.

\section{Acknowledgment}

Supported by National Natural Science Foundation of China (Grant No: 51708236 )

Supported by Fundamental Research Funds for the Central Universities (Grant No: 2016YXMS273 )

\section{References}

1. C.W.Sun, X.P.Lu. Focus on a Center to Solve the Problem of Inpatient Patient's Difficulties in Four Ways [J]. Jiangsu Health Management, 1997(05):32-33. 
2. S.H.Yu. Composite kitchen design based on indoor man-machine environment [J]. Automation and Instrumentation,2014(11):56-58.

3. Y.M.Mou. Research on Kitchen Humanization Design Based on Low-carbon Life [D]. Shenyang Aerospace University,2012.

4. X.Mao. Talking about the Importance of Color Matching in Interior Design [J]. Art Technology,2017,30(12):340.

5. D.Y.Li, B.Chen, Y.F.Li. Overall kitchen manmachine design [J]. Shanxi Architecture,2008(02):45-46.

6. J.H.Li, S.J.Liu. Research on Intelligent Kitchen Design in Information Age [J]. Art Grand View,2017(09):122-123

7. D.W.Zhang. Research on Application of Kitchen Space Structure Design for Affordable Housing [D]. He Bei University,2017.

8. W.C.He. The Development Status and Countermeasures of Sharing Kitchen in Sharing Economy [J]. modern commerce,2017(18):157-158. 